\title{
Politische Kippbildhermeneutik
}

Luca Di Blasi

\section{Einleitung}

Durch das Kippbild wird der Unterschied zwischen "Sehen" und "Sehen als" erkennbar. ${ }^{1}$ Insofern das "Sehen als" der "Auslegung" verwandt ist, ${ }^{2}$ also der Deutung, ergibt sich daraus eine intime Verbindung zwischen Kippbild und Hermeneutik: Kippbildhermeneutik. $^{3}$

Die Anwendung dieser Kippbildhermeneutik auf den Bereich des Politischen scheint dagegen nicht unmittelbar einleuchtend und ist daher erläuterungsbedürftig. Eben eine solche Erläuterung möchte der vorliegende Text vornehmen. Eine Verwandtschaft zwischen Kippbildhermeneutik und Politik scheint sich immerhin schon daraus zu ergeben, dass ein Grundphänomen des Kippbilds, der plötzliche Aspektwechsel, im Bereich zwischen Willkürlichkeit und Unwillkürlichkeit, also zwischen Ohnmacht und Macht - oder präziser: Selbstwirksamkeit - und damit in einem protopolitischen Bereich angesiedelt werden kann.

Das wird schon in den Anfängen der Kippbildforschung deutlich. Der Schweizer Naturforscher Louis Albert Necker, der häufig als wissenschaftlicher »Entdecker» der Kippfigur angesehen wird, ${ }^{4}$ hob 1832 an diesem Phänomen genau das Plötzliche und Unwillkürliche hervor. ${ }^{5}$ Eben dies machte es zu einem Phänomen, denn durch

\footnotetext{
1 Vgl. Ludwig Wittgenstein, Das Blaue Buch. Eine philosophische Betrachtung (Das Braune Buch), Werkausgabe 5, Frankfurt a.M. 1984 sowie ders., Philosophische Untersuchungen/Philosophical Investigations, English and German, trans. G.E.M. Anscombe, Oxford 1953, bes. Teil II, Kap. 11.

2 "Das `Als« macht die Struktur der Ausdrücklichkeit eines Verstandenen aus; es konstituiert die Auslegung." Martin Heidegger, Sein und Zeit (GA Bd. 2), Frankfurt a.M. 1977, 198.

3 Zum Begriff "Kippbildhermeneutik" vgl. auch Luca Di Blasi, Dezentrierungen. Beiträge zur Philosophie der Religion im 20. Jahrhundert, Wien/Berlin 2018, Kap. 1. 4 Allerdings ist die wissenschaftliche Beschäftigung mit dem Kippbild älter. Vgl. Thorsten Jantschek, "Sehen als; Aspektsehen" in: Historisches Wörterbuch der Philosophie, hg. v. Joachim Ritter/Karlfried Gründer, Darmstadt 1996, 162-165, hier 162. 5 "I mean a sudden and involuntary change in the apparent position of a crystal or solid represented in an engraved figure." (Louis Albert Necker, LXI. Observations on
} 
diese Eigenschaften erschien ihm die Kippfigur und veranlasste ihn dazu, sie erklären zu wollen. Für uns ist auch dieser Klärungsversuch selbst aussagekräftig, weil sich in ihm geradezu prototypisch ein neuzeitliches Wissenschaftsverständnis ausdrückt, in dem der Wille zur Erkenntnis und der Wille zur Macht ununterscheidbar ineinander übergehen. Necker sah nämlich als einen Beweis seiner Erklärung des Phänomens ausgerechnet die Transformation der Unwillkürlichkeit der Kippbewegung in eine Willkürlichkeit. ${ }^{6}$ Damit galt ihm als Beweis genau das, was dem Phänomen sein charakteristisches Merkmal, also seine Phänomenalität, seine Weise des In-Erscheinung-getreten-Seins, raubte.

Hier wird erkennbar, dass dem Naturwissenschaftler Necker gar nicht in erster Linie daran gelegen war, dem Phänomen gerecht $z u$ werden, als ihm vielmehr seine provokative Unberechenbarkeit zu rauben und es in eine kontrollierte und willkürliche Bewegung zu überführen. Obwohl gegenüber dem ursprünglichen Phänomen gewaltsam, ist diese Bemächtigung ihrerseits relevant und schreibt sich in das Phänomen der Kippfigur ein: Dass nämlich eine anfänglich kontingente und die eigene Willkür begrenzende Kippbewegung mit der Zeit und durch Übung in eine weitgehend kontrollierte Bewegung überführt werden kann.

Was sich in dieser Ermächtigung zudem andeutet, ist eine kratische, eine vorpolitische Dimension. Diese taucht genau dort auf, wo eine Begrenzung erfahren wird, hier: Die Begrenzung eigener Willkür und Macht, die ein Streben nach Bemächtigung allererst in Gang bringt. Und eben dieser Zusammenhang scheint für eine politische Kippbildhermeneutik relevant: Dass diese nicht nur eine hermeneutische Dimension und das Spiel der Interpretationsmöglichkeiten erkennbar machen kann, sondern auch politische Aspekte, die genau dort sichtbar werden, wo dieses Spiel der Interpretationen auf Grenzen stößt. Diese Begrenzungen werden im Folgenden als potentielle Orte der Politisierung, des Streites gedeutet.

Dabei interessieren mich drei unterschiedliche Begrenzungen und mit ihnen einhergehende Konflikt- und Lösungspotentiale, die mithilfe einer politischen Kippbildhermeneutik herausgearbeitet werden können. Die erste betrifft die sogenannte "Aspektblindheit«,

some remarkable optical phænomena seen in Switzerland; and on an optical phænomenon which occurs on viewing a figure of a crystal or geometrical solid, in: The London, Edinburgh, and Dublin Philosophical Magazine and Journal of Science 1, no. 5 [1832], 329-337, hier 336.)

6 Ebd., 337: »This solution being found, I proved that it was there alone by three different ways. 1st, By being able at my will to see the solid in which position I chose, and to make this position vary at pleasure". 
die Unfähigkeit, etwas als etwas sehen zu können sowie daraus (vorgeblich) erwachsende Konflikte sowie das »Aspektsehenkönnen« als ihre (vorgebliche) Lösung. Die zweite Begrenzung lässt sich daraus entfalten, dass dem Aspektwechsel insofern spezifische Asymmetrien eingeschrieben sind, als nicht beide "Aspekte" von Anfang an als "Aspekte" gesehen wurden. Aus den hier verborgenen Asymmetrien und möglichen Asymmetrieunterstellungen können Konflikte erklärt werden, die auch ein wechselseitiges Bewusstsein der Relativität des eigenen Sehens als "Sehen als" nicht notwendig schlichtet. Eine dritte Begrenzung tritt dort auf, wo relevante oder schwere Zeichen das freie Spiel der Interpretationen unterbrechen. Das gilt auch und gerade für politische Zeichen. Zu Wittgenstein zurückkehrend möchte ich abschließend zu zeigen versuchen, dass der Unterscheidung von "Sehen" und "Sehen als" von Anfang an eine verborgene politische Dimension eingeschrieben war. Im Anschluss an Wittgensteins radikal-selbstaufklärerische Denkbewegung lassen sich eine reduktive Kippbildhermeneutik und ihre politischen Folgen ans Licht bringen.

\section{Reziproke Aspektblindheiten versus Aspekte- sehen-Können}

Wenn Begrenzungen des Spiels der Deutungen hervorragende Orte des potentiellen Konflikts und der Politisierung darstellen, dann sollte sich die sogenannte Aspektblindheit ${ }^{7}$, die Unfähigkeit also, unterschiedliche Aspekte sehen zu können, als eine hervorragende Begrenzung entsprechend in besonderem Maße zur Veranschaulichung politischer Konflikte eignen. Tatsächlich scheint genau dies in einem bekannten Cartoon von Paul Noth aus dem New Yorker von 2014 vorzuliegen:

7 Vgl. dazu Wittgenstein, Philosophische Untersuchungen, 213. 


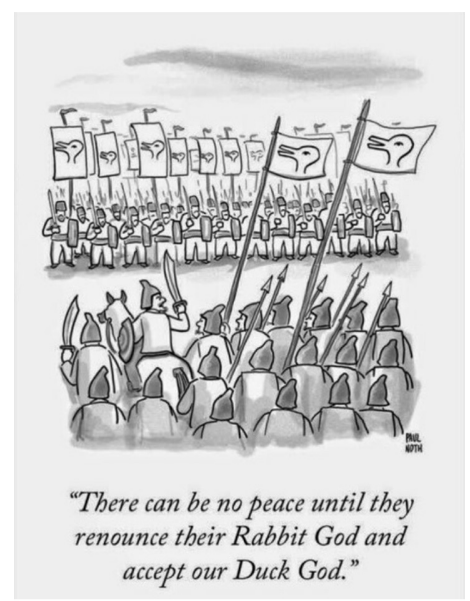

Abb. 1: Paul Noth: "There can be no peace until they renounce their Rabbit God and accept our Duck God ${ }^{8}{ }^{8}$

Das Kippbild dient hier dazu, einen politisch-theologischen Konflikt zwischen zwei Streitparteien darzustellen. Auffällig ist hier die Verbindung zum Feld des Religiösen, man ist verleitet, an mittelalterliche Kreuzritter zu denken und islamische Gotteskrieger und sie als Repräsentanten gegenwärtiger Konflikte zwischen christlichen und islamischen "Fundamentalisten" zu sehen. Beide Seiten scheinen deswegen in Konflikt miteinander zu geraten, weil sie nicht sehen, was der Betrachter sehen kann, dass sie jeweils nur einen Aspekt sehen. Weil sie das nicht sehen, überschätzen sie die Differenz und vergötzen zugleich eine Einseitigkeit und werden dadurch zu intoleranten Gotteskriegern ihres verabsolutierten Aspekts. Damit scheint das Bild in einer langen Aufklärungstradition zu stehen, in der "religiös» schnell mit "dogmatisch" und "fanatisch" gleichgesetzt wurde.

Man kann sogar einen Schritt weiter gehen und hier die kongeniale Illustration der Definition Jacques Rancières vom »Unvernehmen«, von der "mésentente» erkennen:

"Das Unvernehmen ist nicht der Konflikt zwischen dem, der weiß, und jenem, der schwarz sagt. Es ist der Konflikt zwischen

8 Bildzitat aus:The New Yorker (1. Dezember 2014), 78. 
dem, der >weiß< sagt, und jenem, der auch >weiß sagt, aber der keineswegs dasselbe darunter versteht. ${ }^{9}$

Ist das nicht genau das, was die Karikatur auch sagt? Beide Streitparteien sagen Hase-Ente-Kopf (»H-E-Kopf $\left.~^{10}\right)$, haben das auf ihre Fahne geschrieben, aber sie scheinen darunter etwas ganz Verschiedenes zu verstehen. Besteht das Problem, kippbildhermeneutisch und mit Wittgenstein formuliert, also nicht genau darin, dass hier zwei diametral entgegengesetzte Formen der "Aspektblindheit" aufeinanderprallen, wobei die einen nicht in der Lage sind, den Bildgegenstand »Hase«, die anderen den Bildgegenstand "Ente» zu sehen?

Die Spur zur Lösung des Konflikts scheint Paul Noth ebenfalls zu legen, indem er in seinem Cartoon Ludwig Wittgensteins HE-Kopf aus dessen Philosophischen Untersuchungen zitiert und unsere Aufmerksamkeit also auf diesen Text lenkt. Der Aspektwechsel von Kippfiguren diente dem Wiener Philosophen dazu, das "Sehen " vom "Sehen als« zu unterscheiden.

"Ich konnte also den H-E-Kopf von vornherein einfach als Bildhasen sehen. D.h.: Gefragt, >Was ist das? ‘, oder >Was siehst du da?<, hätte ich geantwortet: `Einen Bildhasen`. Hätte man mich weiter gefragt, was das sei, so hätte ich zur Erklärung auf allerlei Hasenbilder, vielleicht auf wirkliche Hasen gezeigt, von dem Leben dieser Tiere geredet, oder sie nachgemacht. Ich hätte auf die Frage >Was siehst du da? nicht geantwortet: >Ich sehe das jetzt als Bildhasen.`[...] Dennoch hätte ein Anderer von mir sagen können ২Er sieht die Figur als Bild-H. «"11

Wohlgemerkt: Auch das "Sehen" sieht einen Aspekt, aber weil dieses Sehen ein stetiges ist, sieht es das nicht, während ein Beobachter zweiter Ordnung, der dem Betrachter des Cartoons zu entsprechen scheint, dieses Sehen als "Sehen als" erkennen kann. Dass unser "Sehen« ein »Sehen als« war, kann uns selbst genau dann erkennbar werden, wenn bei einem Kippbild ein neuer Aspekt aufleuchtet. ${ }^{12}$ Und weil in diesem plötzlichen Aufleuchten ein subjektives Erlebnis oder eine Erfahrung zum Ausdruck kommt, die im stetigen "Sehen" gerade nicht thematisch wird, wird im "Sehen als" eine subjektive Dimension erkennbar, die untrennbare Beziehung zwischen Subjekt und Objekt, Kognition und Perzeption, und eben dadurch

\footnotetext{
9 Jacques Rancière, Das Unvernehmen. Politik und Philosophie, Frankfurt a.M. 2002, 9f.

${ }^{10}$ Vgl. Wittgenstein, Philosophische Untersuchungen, 194.

${ }^{11}$ Ebd., 194f.

12 Vgl. ebd., 194.
} 
kann auch die naive und "fundamentalistische» oder "dogmatische" Identifizierung von Sehen mit "objektiver Wahrnehmung" gelockert werden, ohne dass beide voneinander getrennt werden könnten. Die Verschränkung ist gerade die Bedingung der Möglichkeit, den Aspektwechsel als solchen überhaupt zu erkennen: »Der Ausdruck des Aspektwechsels ist der Ausdruck einer neuen Wahrnehmung, zugleich mit dem Ausdruck der unveränderten Wahrnehmung. ${ }^{13}$

Eine politische Kippbildhermeneutik würde entsprechend den Konflikt der beiden Streitparteien im Cartoon von Paul Noth als Konflikt zweier Aspektblindheiten konzeptualisieren, als Folge zweier Limitierungen, und den Parteien zu zeigen versuchen, dass sie ihr "Sehen als" nicht als solches zu erkennen und damit zu relativieren vermögen, dass sie also nicht erkennen, dass in das, was sie dogmatisch als Sehen eines objektiven Sachverhalts missverstehen, ein Denken und Deuten untrennbar, aber unterscheidbar eingeschrieben ist. Sobald hingegen die Relativierung gelingt, wird der Weg zu einer Bejahung der Diversität frei, die erkennt, dass das andere "Sehen als« das eigene "Sehen als" nicht bedroht, sondern bereichert.

Damit wäre also die Lösung des Konflikts angedeutet. Weder hätte eine Seite recht noch die andere noch auch bestünde die Lösung in einem etwaigen dritten Aspekt. Sie liegt vielmehr darin, dass die Streitparteien einen Aspektwechsel vollziehen und dadurch erkennen, dass sie ihr jeweiliges relatives "Sehen als" mit einer objektiven Wahrnehmung verwechseln und verabsoluierten. Die Lösung scheint hier in der Relativierung der jeweiligen Aspekte zu bestehen, so wie Niels Bohr in einem berühmt gewordenen Vortrag von 1927 (veröffentlicht ein Jahr später) den Welle-Teilchen-Dualismus durch sein Komplementaritätsprinzip zu schlichten versuchte. ${ }^{14}$

Doch ist diese Antwort, bezogen auf den Cartoon, befriedigend? Ein Problem dieser Lösung besteht darin, dass etwas, was sich gerade unwillkürlich vollzieht, die Voraussetzung für die Lösung wäre. Ein willkürlicher Aspektwechsel würde von genau jenen verlangt, bei denen er sich angeblich nicht einmal unwillkürlich ereignet hat. Aber ist der unwillkürliche Aspektwechsel nicht gerade die Voraussetzung dafür, dass dieser Wechsel irgendwann willkürlich vollzogen werden kann? Und wären die Streitparteien daher, gerade wenn sie aspektblind wären, dann nicht auf einen Glauben angewiesen, müssten sie nicht der Kippbildhermeneutikerin blind, weil ohne eigene Sehkraft glauben, die ihnen versichert, sie sähen jeweils nur einen

13 Ebd., 196.

${ }_{14}$ Niels Bohr, The Quantum Postulate and the Recent Development of Atomic Theory, in: Nature 121 (1928), 580-590. 
Aspekt? Und müssten sie nicht gleichzeitig ihrem eigenen klaren und deutlichen Sehen zu misstrauen lernen? Müssten sie also nicht zunächst ein auf den eigenen Augenschein gründendes Wissen durch einen Glauben ersetzen, also zu jenen blind Gläubigen erst werden, als die sie der Betrachter vorschnell abgestempelt hatte, um erst auf dieser Grundlage zu einem komplexeren "Wissen" gelangen zu können?

Aber genauer besehen ist schon die Grundlage der Deutung falsch, die Vorannahme, hier würde der Zusammenprall zweier Aspektblindheiten zum Gegenstand gemacht. Denn der Cartoon geht, von seinem Autor so gewollt oder nicht, über einen solchen selbstzufriedenen Relativismus oder Pluralismus offenbar hinaus. Wenn hier nämlich tatsächlich zwei Formen der komplementären Aspektblindheit vorlägen, wie würden die Armeen in der Lage sein $\mathrm{zu}$ erkennen, dass die anderen unter dem Banner eines anderen Gottes streiten? Würden sie in den Fahnen der anderen nicht notwendig den eigenen Gott wiedererkennen und damit die anderen nicht als Feinde erkennen können? Würde also eine Aspektblindheit nicht sogar verhindern, dass ein Konflikt ausbricht? Und liegt also nicht in der Aspektblindheit das eigentliche Problem, sondern eher in der Annahme, dass die eigene Sichtweise richtig, die andere falsch ist? Und schließt dies nicht die Unterstellung mit ein, der andere sei aspektblind bei gleichzeitiger angemaßter Superiorität des eigenen Aspekte-sehen-Könnens? Ist also die scheinbare Lösung nicht zugleich das eigentliche Problem, und wie ließe sich dieses Problem lösen?

\section{Geronnene Aspekte: Politische Kippbildherme- neutik des faktischen Lebens}

Die hier verborgene Möglichkeit einer Politisierung, die dem Aspekte-sehen-Können zugehört und nicht etwa der Aspektblindheit, möchte ich durch eine weitere Limitation vertiefen, die kippbildhermeneutisch erschlossen werden kann. ${ }^{15}$

Wittgenstein machte, wie wir sahen, auf den Unterschied zwischen dem Sehen des Bildhasen vor und nach dem Aspektwechsel aufmerksam. Vorher sah ich es nicht "als Bildhasen«, sondern ich sah

${ }^{15}$ Ich habe diese Möglichkeit erstmals 2014 vorgestellt. Vgl. Luca Di Blasi, Splitting Images. Understanding Irreversible Fractures Through Aspect Change, in: Multistable Figures. On the Critical Potentials of Ir/Reversible Aspect-Seeing, hg. v. Christoph Holzhey, Wien/Berlin 2014, 67-87. 
"einen Bildhasen", und erst nach dem Aspektwechsel kann ich davon sprechen, dass ich den Bildhasen "als Bildhasen" sehe. Das birgt eine Asymmetrie in sich, die zu einem hermeneutischen Schlüssel für die Relevanz der Zeitlichkeit bzw. der Geschichtlichkeit verwendet werden kann. Die retroaktive Relativierung kann nämlich im Falle von individuellen Lernerfahrungen, Erziehungs-, Sozialisierungsund Kulturalisierungsprozessen nicht einfach dadurch rückgängig gemacht oder ausgelöscht werden, dass ich mir der (Kultur-)Relativität dieser Formationsprozesse bewusst werde. Mit der auf diesem Wege möglichen Vergeschichtlichung einer von Wittgenstein weitgehend ungeschichtlich gedachten Kippbildhermeneutik lassen sich diese und damit auch die mit ihr interpretierbaren Konflikte auf die Ebene des Daseins verlagern.

Selbst wenn sich Arten des "Sehens" als Aspekte erweisen, als bloße Möglichkeiten, Dinge oder Sachverhalte "als etwas « zu sehen, bleiben sie für jene, die in diesem Aspekt sozialisiert wurden, für die Aspekte "Lebensformen", "Kulturen", "Religionen" darstellen, hervorgehoben; selbst dann noch, wenn erkannt und anerkannt wird, dass das für andere nur eine "kulturrelative" und beliebige Möglichkeit ist, etwas in einer gewissen Weise zu sehen oder zu tun. Mit Martin Heidegger formuliert: Der Mensch (das Dasein) ist geworfen (»Faktizität«), er hat sich nicht selbst geboren, nicht selbst hervorgebracht, ist daher auch niemals "existent vor seinem Grunde, sondern je nur aus ihm und als dieser «. ${ }^{16}$

Eine daraus zu entfaltende "politische Kippbildhermeneutik des faktischen Lebens" ermöglicht es zu erkennen, dass Prägungen ("Geworfenheit", "Faktizität») selbst dann wirksam sind, wenn man sie als Prägungen, man könnte auch sagen, als geronnene Aspekte erkennt. Das bedeutet, dass man anzuerkennen hätte, dass Freiheitsräume nur aus dieser Grundlage erwachsen. So wie bei Wittgenstein "Sehen" und "Sehen als" als unterscheidbar, aber untrennbar zusammengehörend gedacht werden, gründen wir existenziell auf Grundlagen, auf Formen des "Sehens", die retroaktiv sowohl als kontingent erkannt werden können, (als Formen des "Sehen als") als auch als nicht vollständig transzendierbar anerkannt werden müssen. Jeder Versuch, diese geronnenen Formen des "Sehen als" als unentrinnbares und von uns unabhängiges "Fundament" setzen zu wollen, geht dabei genauso fehl wie der Glaube, sie ließen sich überspringen.

Was ungeschichtlich-analytisch als Sowohl-als-auch zwischen "Sehen " und "Sehen als" (bzw.: zwischen "Perzeption" und "Kognition", "Sinnlichkeit" und "Denken", "Objekt" und "Subjekt») modelliert

${ }^{16}$ Heidegger, Sein und Zeit, 377. 
werden kann, ${ }^{17}$ kann auf seinshermeneutisch-geschichtlicher Ebene als unaufösliche Verbindung von Faktizität und Existenz rekonzeptualisiert werden, als "geworfener Entwurf«. Damit geht die Anerkennung einher, dass jeder Entwurf auf eine Geworfenheit gründet, die als solche weder einfach konstruiert werden kann noch einfach "gegeben" ist und in diesem Weder-Noch kippbildartigen Charakter hat.

Auf der Grundlage einer solchen Kippbildhermeneutik des faktischen Lebens ist es auch möglich, politische Konflikte der Gegenwart genauer zu beleuchten. Ich möchte das anhand der politisch gerade virulenten Unterscheidung Trans-Cis hier andeuten: ${ }^{18}$ Wenn das Auftauchen der Gender-Kategorie, wie das Auftauchen eines neuen Aspekts, als ein Bruch verstanden werden kann, durch den es retroaktiv möglich wird, Geschlechterrollen vom biologischen Geschlecht zu entkoppeln, dann stellte die Übereinstimmung von "biologischem" und "sozialem" Geschlecht (oder traditioneller formuliert: zwischen "Sein" und "Erscheinung») nur noch eine Möglichkeit dar, nur einen "Aspekt «, den man retroaktiv als "Cis» setzen kann.

"Cis" kann aber, wie schon das Wort irreführend nahelegt, als "Diesseits" und damit als die Stätte einer Spaltung missverstanden werden, als dasjenige, was diesseits von Riss und Spaltung liegt, (kippbildhermeneutisch formuliert: als Vertreter eines vom "Sehen als" isolierbaren objektiven "Sehens"). Als solches erscheint dieses "Cis" als "aspektblind", scheint sich mit etwas "Natürlichem « zu verwechseln und steht hierin diesseits der zahllosen Aspekte, die erst aus dem "Trans" heraus in den Blick geraten und gehört strukturell daher nicht zur Diversität, jener Kette an möglichen Gender-Identitäten.

Versteht sich das "Cis« dagegen als Folge eines "Trans", kann diese Folge unter Verdacht geraten, reaktionär zu sein, bewusste Wahl einer sich retroaktiv als "natürlich" setzenden und daher repressiven und privilegierten Identität, die das Trans ausschließen möchte. Das "Cis» kann also verdächtigt werden, naive Voraussetzung und böse Konsequenz des "Trans" zu sein. In Folge dessen bleibt dem Cis, aus Sicht der Diversität, die sich aus der Spaltung von Gender und Sex ergibt, ein eigener Ort verwehrt und das Cis sieht sich entsprechend einer feindlichen, weil es ausschließenden Diversität gegenüber, so wie

${ }^{17}$ Vgl. auch Sara Fortuna, Wittgensteins Philosophie des Kippbilds. Aspektwechsel, Ethik, Sprache, Wien/Berlin 2012, 44f.

${ }^{18}$ Vgl. dazu ausführlicher Luca Di Blasi, Cis. The Rightist Appropriation of Identity Politics and its Boundaries, in: In Need of a Master. Politics, Theology, and Radical Democracy, hg. v. Dominik Finkelde/Rebekka Klein, Berlin 2021,145-164. 
sich umgekehrt diese als Zusammenschluss von Genderidentitäten sieht, die von einem naiven oder reaktionären "Cis« ausgeschlossen werden.

Genau diese Option kommt, scheint mir, jener im Cartoon gezeigten deutlich näher als die zunächst angenommene zweier reziproker Aspektblindheiten, denn von einer solchen konnte im Cartoon, wie gesagt, nicht die Rede sein: Wie die Betrachterin des Cartoons weiß (zumindest) auch der Heerführer der Armee im Vordergrund ganz genau, dass die anderen unter dem gleichen Banner einen anderen Gott verehren, den H-E-Kopf als etwas anderes sehen. Er ist also hierin durchaus nicht dümmer als die aspektsehende Cartoonbetrachterin. Die offensichtliche Aggressivität geht hier von genau jenem aus, der ohne Zweifel des Aspektwechsels fähig ist.

Vor diesem Hintergrund erscheint Wittgensteins H-E-Kopf auf den Fahnen zweideutiger. Dann ist das mehr als eine bloße Referenz an Wittgenstein und mehr auch als eine Art Paragone, ein Wettkampf zwischen Cartoon und Philosophie. ${ }^{19}$ Es kann auch so gelesen werden, dass uns selbst und gerade die Einsicht in die Aspektualität des eigenen Sehens nicht davor bewahrt, von der anderen Seite als feindlich wahrgenommen zu werden und diese andere Seite selbst als feindlich anzusehen. Selbst wenn man, wie die Postmoderne, mit der Fahne Wittgensteins unterwegs ist, ist man nicht davor gefeit, in unlösbare politische Konflikte miteinander zu geraten - und die wachsenden identitätspolitischen Polarisierungen innerhalb westlicher Gesellschaften scheinen dies zu bestätigen. Liegt also das Problem am Ende bei Wittgenstein?

\section{Politisch-existenzialistische und politisch-reduk- tive Kippbildhermeneutik}

Ich fasse kurz zusammen: Neben der protopolitischen Unwillkürlichkeit des Aspektwechsels als Antrieb einer Bewegung der Selbstermächtigung in der Einführung habe ich zwei Einschränkungen des Aspektwechsels als Schlüssel für die Deutung politischer Konflikte genauer untersucht: Die Aspektblindheit, die als (vorgebliche) Ursache einer konfliktträchtigen Unfähigkeit zum Aspektwechsel und

${ }^{19}$ Der H-E-Kopf Wittgensteins entstammt bekanntlich seinerseits einer Karikatur aus der Zeitschrift Fliegende Blätter, von 1892 (vgl. Fliegende Blätter 97.2465 [1892], 17, http://digi.ub.uni-heidelberg.de/diglit/ fb97/0147; letzter Zugriff: 28.03.2021), so dass wir es bei Noth mit einer Reappropriation einer zuvor vom Philosophen gekaperten Karikatur in eine neue Karikatur zu tun haben. 
zur Selbstrelativierung erschien und damit als ein Problemfall, wobei sich erwies, dass eben diese Deutung vielleicht konfliktträchtiger als die Aspektblindheit selbst ist. Und eine strukturelle Asymmetrie zwischen "Sehen" und "Sehen als«, die als Modell für die Entstehung unterschiedlicherVerdachtshermeneutiken und Konflikte selbst und gerade zwischen Positionen verstanden werden kann, die durchaus zur Selbstrelativierung in der Lage sind.

Davon möchte ich eine weitere Möglichkeit unterscheiden. Sie ergibt sich durch eine existentielle und lebensphilosophische Vertiefung des Aspektsehens, durch die Annahme, dass das "Sehen als" nicht im luftleeren Raum stattfindet, auch nicht nur geschichtliche und kulturelle Voraussetzungen hat, sondern letztlich in der Existenz und im Leben gründet und sich hiervon nicht vollkommen lösen lässt. Auch hierfür bietet Wittgensteins H-E-Kopf indirekt einen Anhaltspunkt.

Um in seinen Philosophischen Untersuchungen den Unterschied zwischen Meldung und Ausdruck eines Erlebnisses zu veranschaulichen, der Meldung (ich sehe) einen Hasen und dem (Gedanken-) Ausdruck: "Ein Hase!«, verlässt Wittgenstein symbolisch die Bildhermeneutik des Bildhasen im Kippbild und geht sozusagen in die Natur hinaus: »Ich sehe eine Landschaft; plötzlich läuft ein Hase vorbei. Ich rufe aus Ein Hase! $\ll^{20}$ Wie bei Dilthey und Heidegger, so verlässt auch hier die Hermeneutik gewissermaßen den Rahmen von Texten und Büchern und expandiert in den Bereich des Lebens. (Es ist interessanterweise die exakt umgekehrte Bewegung zum Naturwissenschaftler Necker, der seinen Text mit Beobachtungen von Naturphänomen in der Schweiz begann und - über die Figur des Kristalls - zur geometrischen Figur des »Neckerwürfels» oder Glaswürfels gelangte. Wittgenstein startet sein Kapitel XI der Philosophischen Untersuchungen mit dem Glaswürfel, leitet von hier zum H-E-Kopf und kommt schließlich zum Hasen in der Landschaft.)

Schon der Ausdruck "Erlebnis" deutet eine Verbindung zum Leben an, und tatsächlich ist dieser Ausruf des Erlebnisses: "Ein Hase!« nicht nur in eine andere Situation eingebettet ("Landschaft"), sondern kann darauf zurückgeführt werden, dass hier eine Bewegung im Raum die unwillkürliche Aufmerksamkeit auf sich gelenkt hatte, so wie die scheinbare Bewegung des Aspektwechsels die Aufmerksamkeit Neckers geweckt hatte. Und dies wiederum kann sich auch darauf zurückführen lassen, dass etwas, das sich bewegt, lebenswichtig sein kann, ebenso wie plötzliche Geräusche in unserer Nähe, die genau deswegen unwillkürlich unsere gespannte Aufmerksamkeit auf sich

${ }^{20}$ Wittgenstein, Philosophische Untersuchungen, 197. 
ziehen. Der Ausruf »ein Hase! « kann dann, je nach Kontext, auch Ausdruck einer Erleichterung sein (es ist nur ein Hase - und keine Schlange oder ein anderes gefährliches Wesen), oder der Freude des Jägers, endlich den Hasen vor der Flinte zu haben.

Von dieser Möglichkeit, dass hinter dem erlebnishaften Aufleuchten eines neuen Aspekts ein zugrunde liegender Instinkt, eine existentielle oder lebenswichtige Dimension erschlossen werden kann, kann eine verwandte Möglichkeit unterschieden werden. Sie besteht darin, dass das Auftauchen eines Aspekts das Spiel der Interpretationen jäh abbrechen kann. Der Fleck auf der CT kann unendlich viele Dinge bedeuten, aber sobald er auch "bösartiger Tumor» bedeuten kann, verändert das freie Spiel der Interpretationen, der Möglichkeiten, etwas als etwas zu sehen, (das ohnehin bereits von der Möglichkeit der Gefahr in Gang gebracht wurde), jäh seinen Charakter. Hier nimmt ein Aspekt Überhand vor allen anderen, er unterbricht das "freie Spiel«, aber nicht deswegen, weil die anderen Möglichkeiten nicht mehr bestünden oder man plötzlich aspekterblindet wäre, sondern weil eine Art des "Sehen als" soviel mehr existentielles Gewicht gewinnt als alle anderen, dass diese gleichsam verblassen und sich die Wahrnehmung auf eine, relevant erscheinende Möglichkeit eingrenzt und fixiert.

Es versteht sich von selbst, dass unzählbare politische Konflikte hier ihre Wurzeln haben: Dass für jemanden ein bestimmtes Zeichen nichts Besonderes bedeutet, während für den anderen das gleiche Zeichen mit Bedrohung und Angst verbunden ist. Selbst wenn beide Seiten erkennen und anerkennen, dass beide Möglichkeiten Formen eines "Sehen als" sind, die beide berechtigt sein können (z.B. dass der Anstieg der Temperaturen auf eine bevorstehende irreversible Erhitzung des Planeten hinweisen kann, aber nicht muss), so bedeutet das nicht, dass für jene, die von dieser Möglichkeit alarmiert sind, der Verweis auf die andere Möglichkeit zu einer Relativierung des eigenen "Sehen als" führen muss.

Oder: Das Hakenkreuz kann auch als uraltes religiöses Symbol gesehen werden, zum Beispiel als Glückssymbol im Hinduismus, Jainismus und Buddhismus. Und es kann gesehen werden als Kennzeichen der NSDAP und später der Flagge des Dritten Reiches. Aber selbst wenn man beides weiß, bedeutet das nicht, dass der Verweis auf diese unterschiedlichen Möglichkeiten geeignet ist, die bedrohliche und daher politisierende Kraft, die von diesem Zeichen für viele ausgeht, zu entschärfen.

Hier scheinen wir uns unmerklich vom Kippbild im engeren Sinne entfernt zu haben, denn die unterschiedlichen Deutungen 
des Hakenkreuzes scheinen nicht mit unterschiedlichen Formen des "Sehen als« einherzugehen. Das "Hakenkreuz» sieht nicht anders aus, wenn ich es so oder so deute. Tatsächlich aber ist dem nicht notwendig so. Bei keinem anderen als bei Ludwig Wittgenstein taucht schon 1930 eine Art Verkippbildlichung des Hakenkreuzes auf, und zwar genau dort, wo er das erste Mal überhaupt das "Sehen als" in terminologischer Weise einführt.

"[V]ielmehr muß dieses rals ein Gesicht sehen verglichen werden $[\ldots]$ mit dem Sehen dieser Zeichnung

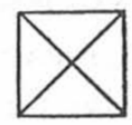

sals ein Quadrat mit Diagonalen oder sals ein Hakenkreuz‘, das heißt, als einen Grenzfall von dieser Zeichnung:

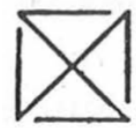

[...] Der Fall [...] ist von besonderem Interesse, weil dieser Ausdruck bedeuten könnte, daß er irgendwie einer optischen Täuschung verfallen ist und glaubt, daß das Quadrat nicht ganz geschlossen ist, daß da Lücken sind, durch die sich das Hakenkreuz von unserer Zeichnung unterscheidet. ${ }^{21}$

Damit hat Wittgenstein eine Figur gefunden, in der von neutralen Möglichkeiten des »Sehen als" (ein zweidimensionales Quadrat mit Diagonalen, eine Pyramide von oben, eine stilisierte Sanduhr etc.) durch minimale Reduktionen an den Ecken eine bestimmte Art abgehoben wird, die, mehr als die anderen, ein erlebnishaftes "Aufleuchten" hervorruft, eben weil dieser Aspekt auf ein Zeichen verweist, das politisch damals, 1930, gerade brisant zu werden begann. Zugleich wird dieses "Sehen als" als defizitär beschrieben, mit einer "optischen Täuschung "verglichen und als ein unvollständiges Sehen (Lücken) betrachtet. Damit aber scheint der Unterscheidung von "Sehen" und "Sehen als" von Anfang an eine politische Subdimension eingeschrieben und eine sich darauf stützende Kippbildhermeneutik wäre also von Anfang an eine politische!

Diese Deutung mag insofern etwas überstürzt erscheinen, als der Kontext eine politische Lesart nicht ohne weiteres nahelegt oder

${ }^{21}$ Wittgenstein, Das Blaue Buch, 253. 
erhärtet. Das aber ändert sich, wenn wir auf den ersten Teil der von 1936 bis 1946 entstandenen Philosophischen Untersuchungen schauen. Auch hier taucht nämlich ein sehr ähnliches Beispiel auf, wobei hier der politische Aspekt kaum noch zu übersehen ist:

"Aber kann ich mir nicht denken, die Menschen um mich her seien Automaten, haben kein Bewußtsein, wenn auch ihre Handlungsweise die gleiche ist, wie immer? - Wenn ich mir's jetzt - allein in meinem Zimmer - vorstelle, sehe ich die Leute mit starrem Blick (etwa wie im [sic!] Trance) ihren Verrichtungen nachgehen - die Idee ist vielleicht ein wenig unheimlich. Aber nun versuch einmal im gewöhnlichen Verkehr, z.B. auf der Straße, an dieser Idee festzuhalten! Sag dir etwa: ‘Die Kinder dort sind bloße Automaten; alle ihre Lebendigkeit ist bloß automatisch.< Und diese Worte werden dir entweder gänzlich nichtssagend werden; oder du wirst in dir etwa eine Art unheimliches Gefühl, oder dergleichen, erzeugen. Einen lebenden Menschen als Automaten sehen, ist analog dem, irgend eine Figur als Grenzfall, oderVariation einer andern zu sehen, z.B. ein Fensterkreuz als Swastika. $\|^{22}$

Auch in diesem Fall muss sich zum "Sehen als" eine spezifische Reduktion hinzugesellen, denn man muss aktiv verhindern, etwas zu sehen, um das Fensterkreuz als Hakenkreuz sehen zu können. Rupert Read hat daraus eine politische Deutungsmöglichkeit abgeleitet:

"Seeing a living human being as an automaton is not simply an error, not simply an upshot of stupidity or cant; nor is it quite a complete and utter existential impossibility, an idle fantasy. It is something that one can lead oneself toward doing. For instance, by bracketing oneself, placing oneself in a position of complete spectatoriality to the reality of others (as philosophers do when they are attempting to consider skepticism about other minds as a live possibility). One might say: the Nazi placed himself as a spectator to the cries of his victims. He didn't truly hear them as cries. He didn't hear them as containing a call to respond to, as manifesting a shared humanity. "23 $^{23}$

Etwas später betont Read, dass »to be able to see one's victims as automata, it helps to see as an automaton sees. $\mathbb{1}^{24}$ Und kann man

${ }^{22}$ Wittgenstein, Philosophische Untersuchungen, 126.

${ }^{23}$ Rupert Read, Wittgenstein's >Philosophical Investigations` as a War Book, in: New Literary History 41 (2010), 593-612, hier 603.

${ }^{24}$ Ebd., 606. 
daher nicht auch sagen, dass es gerade die Nähe ist, in die der Philosoph Wittgenstein zu seinem eigenen Beobachten gerät, die er als unheimlich erfährt? In der Beobachtung dieser "reduktiven Kippbildhermeneutik", wie ich sie nennen möchte, fände, hierin der Rubinvase ähnlich, ${ }^{25}$ ein doppelter Aspektwechsel statt: Nicht nur werden Menschen durch andere Menschen zu Automaten reduziert, also entmenschlicht. Das Unheimliche, das Wittgenstein hier deutlich betont, scheint gerade die Tatsache zu betreffen, dass diese Entmenschlichung jene mitumfasst, die andere Menschen als Automaten sehen. Oder genauer: Gerade indem ich die anderen Menschen als Automaten ansehe, verwandle ich mich (zumindest ihnen gegenüber) zu einem solchen. Die Reduktion betrifft also die Menschen, wie sie demjenigen erscheinen, der sie als Automaten beobachtet, ebenso wie jene, die sie entmenschlichen und die dadurch selbst zu Automaten werden, so wie Fenster- zu Hakenkreuzen werden.

Ein solcher doppelter "Aspektwechsel« könnte ein negatives AhaErlebnis genannt werden. Während das (positive) Aha-Erlebnis darin besteht, dass in der scheinbaren Einfachheit des Sehens plötzlich ein neuer Aspekt, eine Differenz auftaucht, besteht das negative AhaErlebnis genau umgekehrt darin, dass eine scheinbare Differenz verschwindet. Es ist die unheimliche Begegnung mit dem abgewerteten Anderen als den eigenen Doppelgänger, die Ödipus-artige Entdeckung einer schrecklichen Wahrheit, eine Art beschämendes Tat Twam Asi. Im konkreten Kontext ist es der Moment, in dem wir erkennen können, dass unsere Fähigkeit, Aspekte sehen zu können, uns nicht in eine überlegene Position versetzt. Umgekehrt ist genau diese Überlegenheitsannahme der Moment, in dem wir den anderen etwas absprechen, sie reduzieren, und sie gewissermaßen entmenschlichen ("aspektblinde Fundamentlisten", "Automaten", "Nazis" etc.). Es ist, als ob der schmunzelnde Betrachter des Cartoons von Paul Noth, der eben noch seine Überlegenheit gegenüber dem beschränkten und verbohrten religiösen oder politischen Idioten genoss, sich nicht nur selbst plötzlich mitten im Kampfplatz wiederfände, sondern sich selbst als jenen zu erkennen hätte, als den er den anderen gezeichnet hatte, eben weil er ihn als solchen gezeichnet hatte.

Diese Einsicht geht über Bohrs Komplementaritätsprinzip hinaus, wo bereits die Unabhängigkeit der Beobachterposition gegenüber den Phänomenen in Frage gestellt wurde, und auch noch über die Anerkennung der eigenen "exzentrischen Positionalität» (Helmut

\footnotetext{
${ }^{25}$ Di Blasi, Splitting Images, bes. 69-73.
} 
Plessner $\left.{ }^{26}\right)$. Im Anschluss an Wittgenstein lässt sich erkennen, wie eine Beobachterin sich, qua reduktiver Kippbildhermeneutik, als das erweist, was sie bei den anderen zu beobachten meint, sie erkennt sich genau als das, was sie eben noch souverän-jovial belächelt hatte, als dessen Doppelgängerin. Das macht diese Einsicht unheimlich, und eben in diesem negativen Aha-Moment, dem Moment einer unheimlichen Selbsteinsicht, könnte das radikal-(selbst-)aufklärerische Potential einer politischen Kippbildhermeneutik liegen. Und das versetzt diese politische Kippbildhermeneutik selbst in ein kippbildartiges Verhältnis zum Politischen. Das Politische, so könnte man nämlich im Lichte negativer Aha-Erlebnisse sagen, ist eine Maschine, die immer neue Wege findet, andere Gruppen - und damit uns selbst ihnen gegenüber - zu reduzieren. Sie ist eine verlässliche Lieferantin für Optionen, den jeweiligen eigenen Selbstwiederspruch auszulagern. Mit ihrer Hilfe entstehen Relationen, in denen wir lustvoll regredieren, uns dem Todestrieb überlassen können. Negative Aha-Erlebnisse machen uns das deutlich, sie sind Lichtungen an der Grenze der konfliktuellen politischen Maschine - und sie können entsprechend leicht in neue Möglichkeiten der Politisierung eben dieser Einsichten kippen - zum Beispiel, in dem man sie gegen das Politische selbst instrumentalisiert.

- Prof. Dr. Luca Di Blasi unterrichtet Philosophie an der Theologischen Fakultät der Universität Bern, ist assoziiertes Mitglied am ICl Berlin und leitet gegenwärtig das Projekt "Disagreement Between Religions«. Zu seinen Forschungsschwerpunkten zählen Gegenwartsphilosophie und Politische Theologie.

${ }^{26}$ Es fällt in diesem Kontext auf, wie sehr Plessner die für den Menschen charakteristische Exzentrizität mit einer kippbildhermeneutischen Terminologie beschreibt: "Ihm [dem Menschen, LDB] ist der Umschlag vom Sein innerhalb des eigenen Leibes zum Sein außerhalb des Leibes ein unaufhebbarer Doppelaspekt der Existenz, ein wirklicher Bruch seiner Natur." (Helmuth Plessner, Die Stufen des Organischen und der Mensch. Einleitung in die philosophische Anthropologie, Berlin/New York 1975, 292 [Hervorhebungen von LDB].) 